(Aus dem physiologischen Laboratorium der physiko-math. Fakultät der k. Universität Kasan.)

\title{
Über die Latenz der elektrischen Reaktion des Froschherzmuskels bei Doppelreizen.
}

\author{
Nach Versuchen von Herrn J. S. Beritoff mitgeteilt \\ von
}

Prof. A. Samojloff.

(Mit 6 Textfiguren.)

Reizt man einen quergestreiften Skelettmuskel mit zwei rasch aufeinanderfolgenden Reizen, so erscheint, wie neuere Untersuchungen ergeben haben, bei genügend kleinem Reizintervall die elektrische Latenz der Beantwortung des zweiten Reizes länger im Vergleich zu der des ersten Reizes. Wenn über den Grad und über die Deutung der Verspätung des zweiten Aktionsstromes die Ansichten der Autoren differieren, so ist die Tatsache an sich im allgemeinen ausser jedem Zweifel. Natürlich ist es nun zweckmässig, die Frage nach der Reaktion anderer Muskeln und vor allem des Herzmuskels auf Doppelreize in bezug auf die Latenz in Angriff zu nehmen.

Wenn es sich aber um einen relativ so langsam reagierenden Muskel, wie der Herzmuskel ist, handelt, der noch dazu eine sehr stark ausgesprochene refraktäre Periode besitzt, so ist es ganz natürlich, daran zu denken, dass in diesem Falle die Verhältnisse der Latenz bei Doppelreizen einfach auf Grund der mechanischen Herzaktion aufgeklärt werden können. Und in der Tat war die Frage nach der Grösse der mechanischen Latenz in ihrer Abhängigkeit vom Reizintervall resp. von der Kontraktionsphase, auf die der zweite Reizmoment fiel, schon vor vielen Jahren von einigen Autoren experimentell geprüft.

M a re ${ }^{1}$ ) hatte in seiner Arbeit, in welcher er über die Existenz der Refraktärperiode des Froschherzmuskels berichtete, auch die

1) M. Marey, Des excitations artificielles du cour. Travaux du laboratoire du Marey 1876 p. 63 , vgl. p. 73.

Pflüger's Archiv für Physiologie. Bd. 147. 
Frage nach der Latenz der Extrareize berübrt. Er führt hier acht Kurvenreihen vom Froschherzsystolen an; in jeder Kurvenreihe sieht man je einen auf verschiedene Phasen der Kontraktion fallenden Reizmoment des Extrareizes verzeichnet. In den ersten drei Kurvenreihen bleibt der Extrareiz unbeantwortet, weil er das Herz refraktär findet; über die übrigen fünf Reihen wird gesagt: „De la ligne 4 a la ligne 8 , le coeur réagit aux excitations, mais avec des rapidités bien différentes. Ce retard correspond à ce qui $\mathrm{Helm}$ holtz apelle temps perdu pour les muscles volontaires. Or, ce retard va toujours en diminuant à mesure que le coeur est excité dans une phase plus avancée de ca diastole; très-long pour la ligne 4 oì il atteint environ $1 / 2$ seconde, il est presque nul pour la ligne 8." Methodisch ist es von Bedeutung, dass das Herz zwischen den Branchen einer "Pince myographique" elastisch eingeklemmt war, wobei die Branchen einen grossen Teil des Ventrikels und möglicherweise auch die Vorhofventrikelgrenze einnahmen; diese Einrichtung diente zur Reizung des Herzens und gleichzeitig zur Registration der Kontraktionen.

Später wurden ähnliche Versuche von Brunton und $\mathrm{Cash}^{1}$ ) ausgeführt. Diese Autoren konnten bei Anwendung maximaler, auf den Froschventrikel applizierter Reize die Resultate von Marey bestätigen. "A very considerable latency is to be observed in cases where stimulation falls early in the systole. The latency, when this is the position of the shoek, is usually, $5^{6}$ or even more, and oceasionally where stimulation is coincident whit the earliest possible attempt at systole, nearly the whole beat may lapse before reduplication." Es fehlen leider Angaben über den genauen Ort des Ventrikels, an welchen die Elektroden angelegt waren, sowie über die Beschaffenheit der Elektroden, so dass es unklar bleibt, ob die Vorhöfe bei Anwendung maximaler Reize mitgereizt werden konnten. Übrigens wird auch von den Autoren eine Reihe von Versuchen mit minimalen Reizen beschrieben. Das Resultat dieser Reihe lautet allerdings etwas anders: Fällt der Reiz einen Augenblick vor dem Beginn der natürlichen Systole, so ist die Latenz 0,13 Sek., trifft der Reiz etwa die Mitte der Dekreszente, dann dauert die Latenz

1) P. L. Brunton and T. Cash, On the Effect of Elektrical Stimulation of the Frog's Heart. Proceedings of the R. Society of London vol. 35 p. 455, comp. p. 460.1883 . 
0,18 oder 0,2 Sek., und beim Zusammenfallen des Reizes mit dem Gipfel der Systole ist die Latenz 0,2 Sek. Die Dauer der ganzen Ventrikelperiode war in der besprochenen Reihe 1,3 Sek.

Dieselbe Frage wurde darauf in eingehender Weise von Th. Engelmann ${ }^{1}$ ) bearbeitet, wobei er zu anderen Resultaten gelangte, nämlich, dass die Dauer der mechanischen Latenzperiode im ganzen und grossen in keiner Beziehung zur Phase der Kontraktion, auf die der Extrareiz fällt, steht. Die Versuche von Brunton und Cash werden von Engelmann nicht erwähnt. Auf die Ergebnisse von Marey wird dagegen ganz ausführlich eingegangen; Engelmann klärte hierbei die methodologischen Nachteile von Marey's Versuchsanordnung, die den wahren Sachverhalt verdeckten. „Überhaupt hängt nach Marey die Dauer des Latenzstadiums bei jeder Reizstärke von der Phase $a b$, in welche der Reiz fiel, und zwar so, dass sie auch für solche Reize, die während einer Diastole einsetzen, um so länger ist, je weiter vor dem Ende der Diastole letzteres geschieht." „An der Richtigkeit der tatsächlichen Angaben von Marey ist natürlich nicht zu zweifeln, um so weniger, als sie durch Abbildungen von Kardiogrammen belegt sind, welche keine andere Auffassung zuzulassen scheinen, als die ihnen vom Autor gegebene. Irrtümlich nur würde es nach meinen Erfahrungen sein, wenn man meinen wollte, dass es sich hier um ein der Kammermuskulatur überhaupt zukommendes Verhalten handle. Wenn man die Reizung auf die Herzspitze beschränkt, sei es nun, dass man an der abgeschnittenen oder abgebundenen Kammerspitze experimentiert, orler mit solchen Stromstärken und in solcher Entfernung von der Kammervorkammergrenze zeigt, dass die hier gelegenen Teile (Atrien, Bulbus, Kammerbasis) nicht direkt erregt werden können, so finde ich ausnahmslos folgendes, von Marey's Befunden abweichendes Verhalten. Gleichviel in welche Phase der Reiz fällt und welches seine Stärke ist, wenn er überhaupt Erfolg, d. h. eine $V_{s}$ zur Folge hat, so tritt dieser sogleich ein, d. h. nach einem sehr kurzen Latenzstadium, durchschnittlich etwa nach 0,1 Sek." Die Ursache für die abweichenden Resultate Marey's findet Engelmann in folgendem: "Ich glaube sie darin finden zu müssen, dass Marey's Versuchseinrichtung eine Reizung der Ventrikelbasis und

1) Th. Engelmann, Refraktäre Phase und kompensatorische Ruhe. Arch. f. d. ges. Physiol. Bd. 59 S. 309, vgl. S. 318-319. 1895. 
der angrenzenden Teile (Atrium und Bulbus) nicht ausschloss. Es war ja auch Marey gar nicht um isolierte Reizung der Herzspitze zu tun. Da die Arme des Herzmyographs, welche das blossgelegte Herz zwischen sich klemmten, bei M arey zugleich als Elektroden dienten und, nach Beschreibung und Zeichnung zu urteilen, die an die Basis grenzende Partie des Ventrikels zwischen sich fassten, müssen schon schwache elektrische Ströme alle an der AV-Grenze zusammenstossenden Herzabschnitte getroffen haben. Und so ist die Vermutung begründet, dass die von $\mathrm{M}$ a r ey künstlich hervorgerufenen, Systolen in jenen Fällen längerer Latenz nicht einer direkten Erregung der Muskulatur der Herzspitze, sondern indirekter Reizung ihren Ursprung verdankten ..."

Was nun die Frage nach der elektrischen Latenz im besprochenen Sinne anbetrifft, so hat wohl Keith Lucas ${ }^{1}$ ) als erster dieselbe aufgestellt und in einigen besonders dazu angeführten Versuchsreihen geprüft. Wie ich ${ }^{2}$ ) es schon in einer anderen Stelle hervorhob scheint leider in den Lucas'schen Versuchen vermutlich ein Missverständnis mit untergelaufen zu sein.

K. Lucas hat nämlich erstens einen Versuch mit Doppelreizungen der Ventrikelbasis oder des Vorhofes am nach der zweiten Stannius'schen Ligatur stillstebenden Froschherzen vorgenommen und dabei die vom Ventrikel abgeleiteten Aktionsströme mit dem Kapillarelektrometer registriert. Es stellte sich heraus, dass, anstatt der anfänglichen Latenz 0,037 Sek., die Latenz nach dem zweiten Rejze des Doppelreizes bis 0,33 Sek. betrug, d. h. die Latenz war infolge des kurzen Reizintervalles auf etwa das Zehnfache gestiegen.

In einem zweiten Versuch wurden bei derselben Ableitung der Ströme des Ventrikels eines spontan klopfenden Froschherzens Extrasystolen in verschiedenen Phasen der Ventrikelsystole durch Einzelreize der Ventrikelbasis hervorgerufen. Wenn der Reiz sofort nach Ablauf der Refraktärperiode appliziert war; so vergingen zwischen dem Reiz und der elektrischen Beantwortung 0,94 Sek.; reizte man später, so war die Zeitdauer kürzer und schliesslich 0,56 Sek.

1) K. Lucas, The electric vesponse of the gastrocnemius muscle of the frog to two stimuli. The Journ. of Physiol. vol. 41 p. 308, comp. p. 368. 1910.

2) A. Samojloff, Über die Verspätung des zweiten Aktionsstromes bei Doppelreizungen des quergestreiften Muskels. Arch. f. d. ges. Physiol. Bd. 143 S. 453 , vgl. S. 489 . 1912 . 
Der dritte Versuch ist in derselben Weise ausgeführt mit dem Unterschiede, dass jetzt der Vorhof gereizt wurde. Die latente Periode des elektrischen Effektes dauerte jetzt genau so lange wie im zweiten Versuch; die Zeit zwischen Extrareiz und Extraeffekt schwankt je nachdem, auf welchen Moment der ersten Schwankung der Reiz fällt, zwischen 0,91 Sek. und 0,55 Sek.

Es war für mich früher und es ist mir auch jetzt, nachdem ich mich durch eigene Versuche überzeugen konnte, dass in der Tat der zweite Reiz bei einem kurzen Doppelreize eine bedeutend verspätete elektrische Aktion bewirkt, vieles in den Ergebnissen von Keith Luc a s unklar. Erstens ist es zu verwundern, dass im ersten Versuch die Verspätung der zweiten Schwankung 10 mal so gross ist wie die Latenz der ersten Schwankung, wogegen die Verspätung in den Versuchen 2 und 3 nicht einmal den doppelten Betrag der kürzesten Latenz beträgt. Auf der Lucas'schen Aufnahme vom ersten Versuche fehlen die Kontraktionskurven; da wir aber wissen, dass die Dauer der Kontraktion des Ventrikels nicht bedentend länger ist wie die der Schwankung, so lässt sich annehmen, dass im Versuch 1 der zweite Reiz so gut wie zu Ende der Systole appliziert war - um so weniger einleuchtend ist es, weshalb die Verspätung einen derartigen Betrag erreichen konnte.

Weiter ist es unverständlich, warum einmal die kürzeste Latenz (im ersten Versuch) 0,037 Sek. und ein anderes Mal (in den Versuchen 2 und 3) über eine halbe Sek. dauert; die kürzeste Latenz ist also in den Versuchen 2 und 3 etwa $16 \mathrm{mal}$ so gross wie im Versuch 1.

Ganz merkwürdig und entschieden auf einem Versehen beruhend ist weiter das Ergebnis, nach welchem die Zahlen für die kürzeste sowohl wie für die längste Latenzdauer identisch im zweiten und dritten Versuch ausfallen, obwohl man in dem einen Versuch den Vorhof, im anderen den Ventrikel gereizt hat.

Es war augenscheinlich eine Mitreizung des Vorhofes bei Applikation des Reizes auf den Ventrikel bei Lucas wie nach Engelmann's Erklärung in Marey's Versuchen zustande gekommen.

Die kritische Besprechung der erwähnten Versuche von K. Lu c a s habe ich in der zitierten Arbeit mit folgenden Worten geschlossen: "Ich will mit dem Obigen nicht sagen, dass beim Herzmuskel jegliche Verspätung im definierten Sinne überhaupt fehlt. Meine eigenen Versuche, die dieses Thema berühren, waren zu einem ganz anderen 
Zwecke unternommen und sind auch nicht gross genug an Zahl, um diese Frage $\mathrm{zu}$ entscheiden. Die Frage nach der Verspätung der elektrischen Reaktion des zweiten Reizes bei Doppelreizen seitens des Herzmuskels hat ein gewisses prinzipielles Interesse, und es wäre in der Tat wünschenswert, dieselbe noch einmal einer exakten und allseitigen Prüfung zu unterwerfen."

Ich veranlasste daher Herrn J. S. Beritoff, eine Reihe von Versuchen über die Latenz der Aktionsströme des Froschherzens bei Änderung der Reizdistanz zu unternehmen.

Der einfachste Versuchsplan zur Prüfung der obigen Frage bestand darin, einen im Stannius'schen Stillstande sich befindenden Froschherzapparat zunächst nicht mit Doppelreizen von bestimmtem Reizintervall, sondern überhaupt mit einer Reihe von Reizen von verschiedenem Intervall zur Tätigkeit anzuregen; aus den Ventrikelelektrogrammen, die also mit zufälliger Zeitdistanz aufeinanderfolgten, sollte dann die Beziehung zwischen Reizdistanz und elektrischer Latenz hervortreten. Nach diesem Schema wurden mehrere Reihen von Versuchen angestellt, die auch zu bestimmten Ergebnissen führten.

Was nun die Methodik anbetrifft, so sei in aller Kürze folgendes erwähnt. Das in situ befindliche Herz eines dekapitierten Frosches wurde an der Spitze suspendiert, wobei die Suspensionsklemme mit dem Hebel nicht vermittelst eines Fadens, sondern vermittelst eines an den Haken angelöteten sehr feinen Drahtes verbunden war. Eine zweite ähnliche Klemme mit einem angelöteten Draht fasste die Ventrikelspitze in der nächsten Nähe von der ersten Klemme. Die beiden Klemmen stellten die Reizelektroden dar und wurden mit feiner Gummiröhren in einen schon früher beschriebenen Weise umgeben ${ }^{1}$ ). Die eine ableitende Elektrode war in einiger Entfernung von den Reizelektroden in Form eines die Spitze im ganzen Umfange umschlungenen Fadens angelegt; die zweite Elektrode, ebenfalls ein Faden, war um den Vorhof geschlungen. Die Reize erzengte man durch Unterbrechung des primären Stromes vermittelst des Engelmann'schen Polyrheotoms. Die Scheibe des letzteren wurde mit der Hand gedreht; eine ganze Umdrehung er-

1) A. Samojloff, Weitere Beiträge zur Elektrophysiologie des Herzens. Arch. f. d. ges. Physiol. Bd. 135 S. 417, vgl. S. 421. 1910. 
zeugte eine einzige Unterbrechung; der Schliessungsinduktionsstrom wurde in bekannter Weise durch eine in entsprechendem Zeitpunkt ausgeführte Nebenschliessung des sekundären Kreises im Polyrheotom vom Froschherz abgeblendet.

Die Aktionsströme wurden vermittelst des Saitengalvanometers (Edelmann's grosses Modell) unter 800 maliger Vergrösserung (Apochromat Zeiss 8,0 mm, Projektionsokular Zeiss 4) registriert; gleichzeitig mit den Saitenausschlägen kamen die Bewegungen des Suspensionshebels, des Reizmarkierers und der Stimmgabel (25 Schwingungen in 1 Sek.) zur Registration. Das lichtempfindliche Papier auf der Trommel war $13 \times 64 \mathrm{~cm}$ gross; bei einer Trommelumdrehung wurde nur ein dritter Teil der Höhe ausgenutzt, so dass man auf demselben Papier drei Serienaufnahmen machen konnte. Die Schnelligkeit der Umdrehung war etwa $3,0 \mathrm{~cm}$ pro 1 Sek., so dass auf dem ganzen Papierstreifen in allen drei Reihen zusammen etwa 20 Systolenaufnahmen Platz finden konnten.

Natürlich kam es darauf an, mit dem Reize am tätigen Herzen nicht zu spät zu kommen, um die möglichst günstigen Bedingungen für das Ausbilden einer eventuellen Verspätung des Aktionsstromes zu schaffen. Nach meiner früheren Erfahrung mit Doppelreizen am Froschherzen hat ein nicht mittelstarker Reiz am tätigen Herzen erst dann Erfolg, wenn er etwa am Ende der elektrischen Schwankung der vorhergehenden Systole appliziert wird ${ }^{1}$ ). Es schien mit anderen Worten, dass die refraktäre Periode im allgemeinen erst nach $\mathbf{A b}$ lauf der $T$-Zacke zu Ende kommt.

Schon unsere ersten orientierenden Versuche zeigten num aber mit vollkommener Konstanz, dass, wenn der zweite Reiz den Ventrikel zu einer Zeit trifft, wo der Aktionsstrom auf den ersten Reiz bereits ganz abgelaufen ist, eine Verspätung des entsprechenden zweiten Aktionsstromes entweder vollkommen fehlt, oder, was seltener vorkommt, bloss ganz unbedeutend erscheint. Um möglichst früh den Reiz mit positivem Erfolg am tätigen Herzen applizieren zu können, blieb nichts anderes übrig, als den Reiz stärker zu wählen. Man kann sich leicht überzeugen, dass ein stärkerer Reiz auch während der Periode der $T$-Zacke, namentlich während der Dauer der Dekreszente derselben, sich wirksam erweist; sehr starke Schläge erregen den Ventrikel auch dann, wenn sie im Momente des Gipfels

1) A. Samojloff, l. c. S. 450 . 
der T-Zacke oder zuweilen sogar auch noch früher, während der Dauer des ersten Teils der T-Zacke, den Herzmuskel treffen. Nun stellt sich aber dabei folgendes heraus. Wendet man stärkere Reize an, so macht sich deutlich eine Komplikation bemerkbar, die darin besteht, dass ein ein stillstehendes Herz treffender Reiz um so früher eine elektrische Reaktion bewirkt, je stärker er ist. Die elektrische Latenz erweist sich somit in ihrer Dauer von der Stärke des Reizes abhängig. Nachdem diese Erscheinung zuerst an der Hand unserer ersten Kurvenreihen bemerkt wurde, war es wünschenswert, dieselbe in extra dazu angestellten Versuchen zu prüfen.

Es sei hier ein derartiger Versuch, den wir der Besprechung der unser Hauptthema berührenden Kurvenreihen vorausschicken möchten, angeführt. Die Trommel öffnet während ibrer Umdrehung einen Kontakt und erzeugt auf diese Weise einen Induktionsschlag, welcher ein im Stannius'schen Stillstande befindliches Herz trifft. Aktionsstrom, Kontraktionskurve, Zeitkurve (0,04 Sek.), Reizsignal wurden verzeichnet. Auf der Fig. 1 sieht man das Resultat dreier in Pausen von mehreren Minuten vorgenommener Reizungen, wobei bei $B$ der Reiz stärker war wie bei $A$ und bei $C$ stärker wie bei $B$. Die Berechnung ergibt, dass die elektrische Latenzdauer in $A-0,056$ Sek., in $B-0,04$ Sek. und in $C 0,024$ Sek. beträgt (die Abstände der Spiralen waren in $A, B$ und $C-10,5 \mathrm{~cm}, 8,5 \mathrm{~cm}, 6,0 \mathrm{~cm})$. Durch blosse Änderung der Reizstärke lässt sich also die Latenzdauer um mehr als zweimal ändern.

Aus der Fig. 1 ist zu ersehen, dass mit Verstärkung des Reizes die Stärke der ins Galvanometer hereinbrechenden Schleifen in hohem Grade zunimmt, so dass man vor allem die Verkleinerung der Latenzdauer auf die durch Stromschleifen bewirkte Reizung der von den Reizelektroden entfernteren und den Ableitungselektroden näher gelegenen Stelle des Herzens zurückführen muss. Anderseits könnte man auch an die viel diskutierte Möglichkeit denken, dass ein starker Reiz schneller geleitet wird. Wir wollen aber nicht weiter auf den Grund der beschriebenen Erscheinung eingehen und nehmen die Tatsache als solche an.

Wenn wir früher erklärten, weshalb es nötig ist, bei Versuchen über die Abhängigkeit der Latenz von der Reizdistanz Reize von erheblicher Stärke anzuwenden, so kommen wir jetzt auf Grund der eben beschriebenen Beziehung der Latenz zur Reizstärke zum Schluss, dass anderseits die Stärke der Reize nicht zu weit getrieben werden 
darf. Wird der Reiz noch stärker wie in Fig. $1 A$, so werden die Schleifenzacken noch stärker, die Latenz augenscheinlich noch kleiner, und man kann überhaupt nur mit Mühe entscheiden; ob irgendeine Latenz vornanden ist. Wir mussten also in unseren Versuchen bei der Wahl der Reizstärke uns von den auseinandergesetzten Gesichtspunkten leiten lassen.

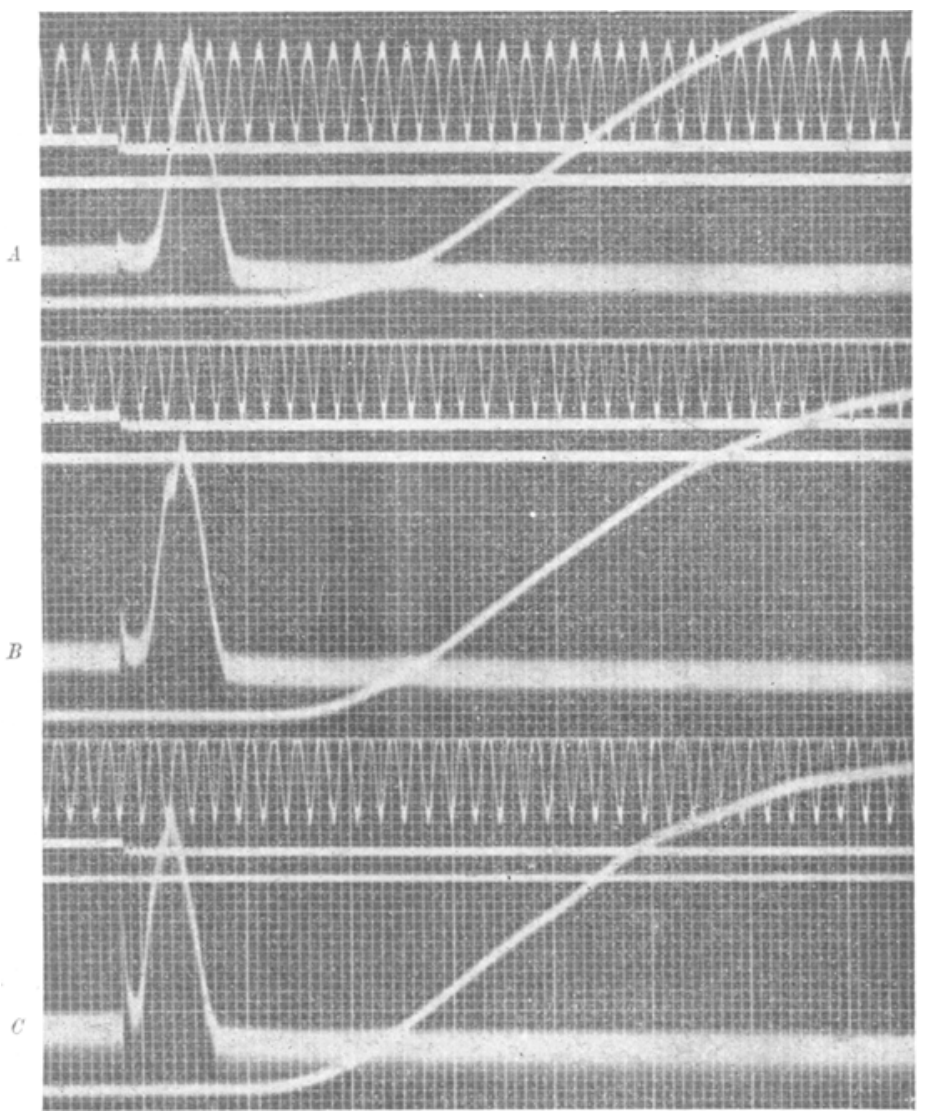

Fig. 1. Drei Ventrikelelektrogramme nach Reizung der Ventrikelspitze mit von $A$ bis $C$ zunehmender Reizstärke. Zeitmarken in 0,04 Sek.

Was nun die Versuchsreihen mit Reizen, die das Herz in verschiedenen Phasen seiner Tätigkeit darstellen, betreffen, so stellte es sich heraus, dass in der Tat eine ganz deutliche Beziehung zwischen der Phase, in welcher das Herz gereizt wird, und der elektrischen Latenzdauer existiert, und zwar je früher der Reiz nach Abschluss der refraktären Periode fällt, desto länger wird die Latenz. 
Wir führen eine von den vielen Versuchsreihen mit Angabe sämtlicher Daten an. Die Reihe enthält über 30 Reizungen. Man bemühte sich im allgemeinen, die Reizungen so vorzunehmen, dass grosse Reizdistanzen mit kleinen abwechselten, um auf diese Weise eine Annäherung an Doppelreize mit kleinem Reizintervall zu erzielen. Es erwies sich aber, dass das in der angegebenen Weise nicht zu erreichen war, denn die Dauer der Herzperiode hängt in so hohem Grade vom Reizintervall ab, dass man beim Abmessen eines grossen resp. kleinen Intervalls sofort in die Hände des Zufalls fällt.

Wir betrachten deshalb unsere Reihe als eine fortlaufende Reihe von Reizungen und geben in der Tabelle I nach Möglichkeit sämtliche Data, die die Beziehung der elekt.ischen Latenz zu anderen Momenten illustrieren, wieder.

Tabelle I.

\begin{tabular}{|c|c|c|c|c|c|}
\hline $\begin{array}{c}\text { Nummer } \\
\text { des } \\
\text { Ver- } \\
\text { suchs }\end{array}$ & $\begin{array}{c}\text { Elektrische } \\
\text { latente } \\
\text { Periode }\end{array}$ & $\begin{array}{c}\text { Zeitintervall } \\
\text { zwischen dem } \\
\text { zweiten } \\
\text { Reizmoment } \\
\text { und dem Gipfel } T \\
\text { der vorhergehen- } \\
\text { den V. E. G. }\end{array}$ & $\begin{array}{l}\text { Die Dauer } \\
\text { des V.E.G. }\end{array}$ & $\begin{array}{c}\text { Die } \\
\text { Reizdistan } z\end{array}$ & $\begin{array}{c}\text { Die } \\
\text { mechanische } \\
\text { Latenz- } \\
\text { periode }\end{array}$ \\
\hline $\begin{array}{r}1 \\
2 \\
3 \\
4 \\
5 \\
6 \\
7 \\
8 \\
9 \\
10\end{array}$ & $\begin{array}{l}0,024 \\
0,035 \\
0,025 \\
0,034 \\
0,025 \\
0,034 \\
0,027 \\
0,030 \\
0,025 \\
0,05\end{array}$ & $\begin{array}{l}1,0 \\
0,13 \\
2,08 \\
0,20 \\
2,50 \\
0,24 \\
1,64 \\
0,36 \\
1,96 \\
0,10\end{array}$ & $\begin{array}{l}2,2 \\
1,56 \\
2,16 \\
1,45 \\
2,13 \\
1,48 \\
2,0 \\
1,52 \\
2,0 \\
1,28\end{array}$ & $\begin{array}{l}2,10 \\
2,30 \\
3,30 \\
2,20 \\
3,90 \\
2,16 \\
2,45 \\
2,16 \\
3,36 \\
1,92\end{array}$ & $\begin{array}{l}0,30 \\
0,26 \\
0,38 \\
0,40 \\
0,34 \\
0,30 \\
0,26 \\
0,20 \\
0,28 \\
0,34\end{array}$ \\
\hline $\begin{array}{l}1 \\
2 \\
3 \\
4 \\
5 \\
6 \\
7 \\
8 \\
9\end{array}$ & $\begin{array}{l}0,063 \\
0,025 \\
0,04 \\
0,022 \\
0,033 \\
0,023 \\
0,032 \\
0,030 \\
0,066\end{array}$ & $\begin{array}{l}0,05 \\
2,0 \\
0,08 \\
1,72 \\
0,24 \\
1,44 \\
0,69 \\
0,72 \\
0,05\end{array}$ & $\begin{array}{l}1,12 \\
2,0 \\
1,36 \\
2,05 \\
1,4 \\
1,92 \\
1,62 \\
1,60 \\
1,2\end{array}$ & $\begin{array}{l}2,0 \\
3,6 \\
2,0 \\
3,0 \\
2,0 \\
2,7 \\
2,3 \\
2,22 \\
1,6\end{array}$ & $\begin{array}{l}0,22 \\
0,34 \\
0,26 \\
0,26 \\
0,32 \\
0,30 \\
0,30 \\
0,24 \\
0,36\end{array}$ \\
\hline $\begin{array}{r}1 \\
2 \\
3 \\
4 \\
5 \\
6 \\
7 \\
8 \\
9 \\
10 \\
11\end{array}$ & $\begin{array}{l}0,030 \\
0,028 \\
0,022 \\
0,024 \\
0,055 \\
0,023 \\
0,033 \\
0,037 \\
0,05 \\
0,026 \\
0,037\end{array}$ & $\begin{array}{l}0,28 \\
1,68 \\
0,76 \\
1,52 \\
0,05 \\
1,8 \\
0,18 \\
1,2 \\
0,10 \\
1,8 \\
0,36\end{array}$ & $\begin{array}{l}1,68 \\
2,00 \\
1,82 \\
1,90 \\
1,20 \\
2,0 \\
1,30 \\
1,7 \\
1,2 \\
1,8 \\
1,4\end{array}$ & $\begin{array}{l}\overline{3,0} \\
2,66 \\
3,0 \\
1,80 \\
2,80 \\
2,18 \\
2,5 \\
1,8 \\
2,8 \\
2,0\end{array}$ & $\begin{array}{l}0,30 \\
0,28 \\
0,28 \\
0,32 \\
0,22 \\
0,28 \\
0,22 \\
0,25 \\
0,22 \\
0,28 \\
0,30\end{array}$ \\
\hline
\end{tabular}


Die angegebenen Zahlen stammen von einem und demselben Versuch, in welchem drei Reihen in der eingangs beschriebenen Weise eine nach der anderen aufgenommen waren. Um die Beziehung der Latenzdauer zu der Phase der Herztätigkeit klarer darzustellen, ist es zweckmässig, die angeführten Zahlen in eine Graphik einzutragen. Es fragt sich nun, welche Zahlenreihe ausser der der Latenzdauer wir in die Graphik aufzunehmen haben. Hätten wir es mit echten Doppelreizen zu tun, so würde es am einfachsten sein, die Latenzdauer mit der Reizdistanz in ihrer Abhängigkeit graphisch darzustellen, um den Einfluss der Phase der Herztätigkeit auf die Latenz zu übersehen. Da aber in unserem Fall, wie oben auseinandergesetzt wurde, wir vielmehr eine fortlaufende Reihe von Reizungen vor uns haben, und da anderseits die Herzperiode in hohem Grade bezüglich ihrer Dauer von der Reizperiode abhängt, so definiert das Reizintervall an sich nur wenig die Phase der Herztätigkeit. Es ist leicht einzusehen, dass irgendein und dasselbe Reizintervall einmal (nach mehreren rasch aufeinanderfolgenden Reizungen) sich als ein Intervall von relativ langer Dauer, ein anderes Mal (nach mehreren weit voneinander abstehenden Reizen) als ein sehr kurzes Reizintervall erweisen kann. Deshalb scheint es zweckmässiger, nicht die Reizdauer, d. h. die Dauer zwischen dem ersten und dem zweiten Reizmoment, zu nehmen, sondern die zwischen einem gut definierbaren Zeitpunkt im Ventrikelelektrogramm (z. B. den Gipfel der T-Spitze) nach dem ersten (d. h. dem vorhergehenden) Reiz und dem zweiten (d. h. dem in Frage stehenden) zur Definition der Phase der Herztätigkeit zu wählen. In der dritten Kolonne sind gerade diese Werte angegeben. Selbstredend ist die eben definierte Grösse nur dann zu gebrauchen, wenn der zweite Reiz nicht vor dem Gipfel $T$ des vorhergehenden V.E.G. fällt. Das trifft gerade in der in Rede stehenden Reibe $\mathrm{zu}$, wenn auch allerdings einige Reize sehr nahe vom Gipfel $T$ fallen.

In der Graphik Fig. 2 sind die Werte der elektrischen Latenzdauer auf der Ordinatenachse und die Werte der Kolonne 3 auf der Abszissenachse aufgetragen.

Da, wie erwähnt, die Dauer der ganzen Schwankung V.E.G. immer kleiner wird, wenn der Reiz den Ventrikel sukzessive früher nach Ablauf der Refraktärperiode fällt, so führen wir in der Graphik Fig. 3 die Abhängigkeit der Latenzdauer (Ordinate) und V.E.G. Dauer (Abszisse) der zweiten Schwankung an. 
Die beiden Figuren zeigen deutlich, dass im allgemeinen eine Beziehung zwischen elektrischer Latenzdauer und Tätigkeitsphase, in die der Reiz fällt, ausser jedem Zweifel steht. Ein in bestimmten Grenzen früher einfallender Reiz bewirkt eine längere Latenz. Da

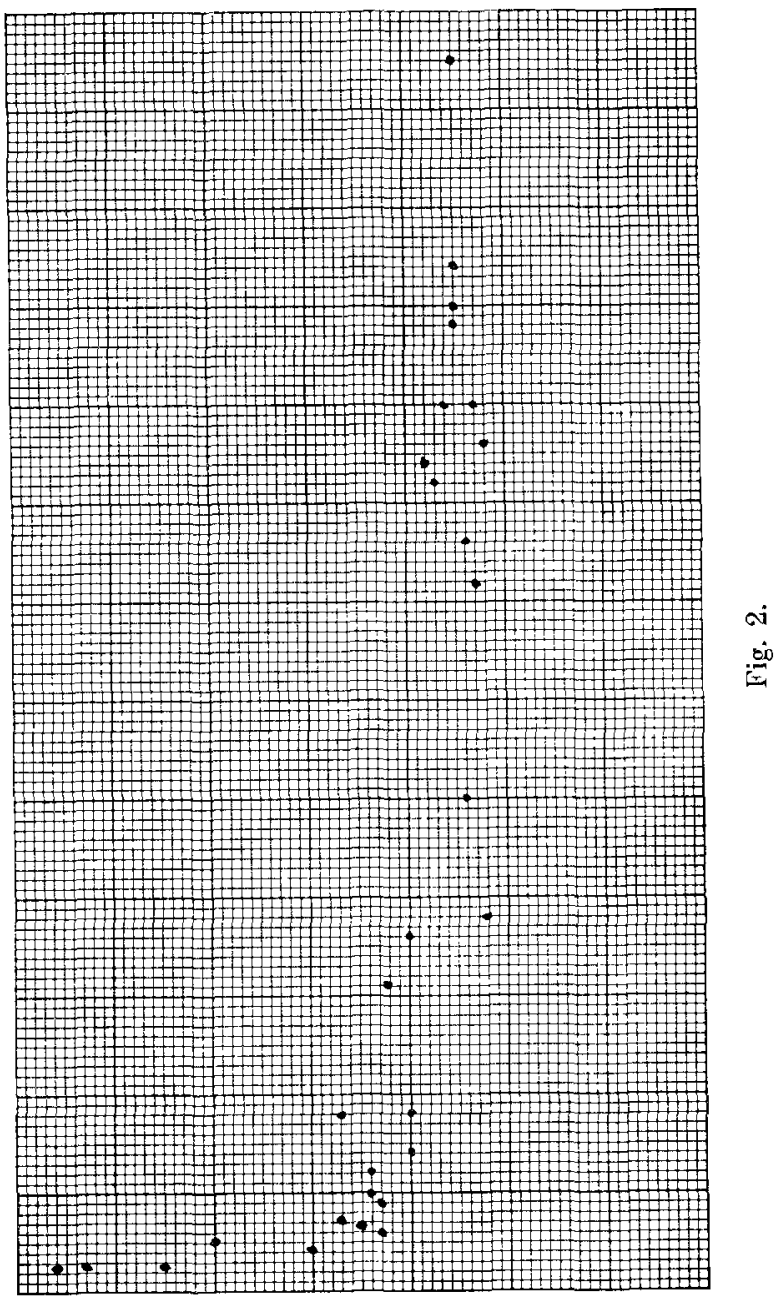

aber ausserdem der ceteris paribus früher einfallende Reiz auch eine kleinere Dauer der ganzen Schwankung zur Folge hat, so lässt sich der Sachverhalt auch in der Weise formulieren, dass in gewissen Grenzen einer Herzaktion mit einer kleineren Schwankungsdauer eine längere elektrische Latenz entspricht. 
Im besprochenen Versuch ist die kleinste Latenz etwa zwei- bis dreimal so klein wie die grösste Latenz.

Bei dieser Gelegenheit konnte man auch sehr gut die Angaben von Engelmann prüfen, dass die mechanische Latenz unabhängig davon ist, wie spät nach Beendigung der refraktären Periode der neue Reiz das Herz trifft. Wie wir früher sahen, ist nach Engelman $n$ die Latenzỏauer die gleiche, ob der Reiz spät oder früh fällt. Unsere Zablen der mechanischen Latenz (Kolonne 6 der Tabelle I) weisen überhaupt bedeutendere Abweichungen voneinander auf, wie die Zahlen von $\mathrm{E} n \mathrm{~g}$ e I -

$\mathrm{m}$ a n n. Am grössten sind die Abweichungen bei ganz kleinen Reizintervallen, was damit zusammenhängen mag, dass bei kleinen Reizintervallen, wenn also die zweite Kontraktion nicht von der Nullinie beginnt, die Ausmessung der mechanischen Latenz mit grösseren Fehlern behaftet ist. Jedenfalls konnte man eine allgemeine Steigerung der Latenzwerte der mecha-

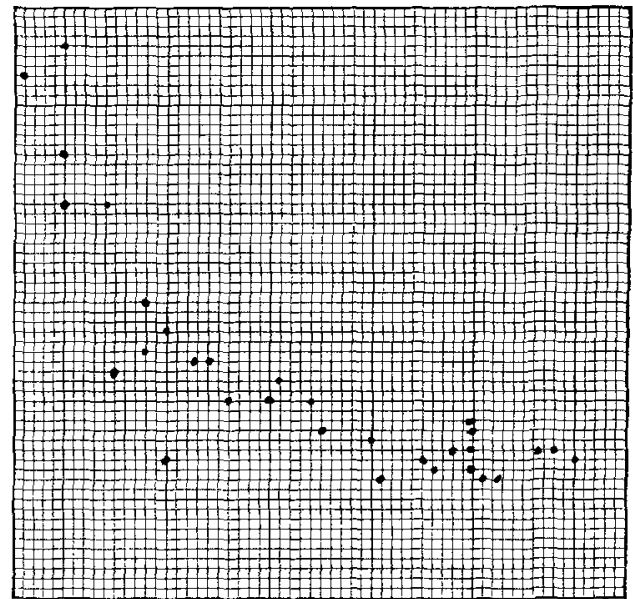

Fig. 3. nischen Herzaktion bei früher einfallenden Reizen nicht feststellen, und wohl in diesem Sinne können wir die Behauptung von Engelman $n$ bestätigen.

Dass die Punkte in Fig. 2 und 3 so zerstreut liegen und sich nicht dem Verlaufe einer Kurve fügen, kann man möglicherweise darauf zurückführen, dass wir es bei unserer Versuchsanordnung ausser dem beabsichtigten Faktor noch mit anderen Faktoren, deren Konstanz nicht genug gesichert ist, zu tun haben. Es liess sich am ehesten vermuten, dass die Latenzdauer in ihrer Abhängigkeit von der Tätigkeitsphase des Herzens sich exakter wird bestimmen lassen, wenn man das Herz mit echten Doppelreizen reizen würde.

Es wurden deshalb auch in dieser Weise mehrere Versuchsreihen ausgeführt. 
Um schärfer die Ausmessung der elektrischen Latenz ausführen zu können, wurde in diesen Versuchen die Trommel mit grösserer Geschwindigkeit in Rotation versetzt (etwa $7-8 \mathrm{~cm}$ in 1 Sek.). Bei dieser Schnelligkeit der Bewegung konnten auf dem ganzen Umfang der Trommel, auf dem $64 \mathrm{~cm}$ langen Papierstück, überhaupt nur zwei Schwankungen entsprechend einem einzigen Doppelreiz Platz finden. Auf jedem Stück Papier (13 cm breit) wurden drei Aufnahmen, eine unter der anderen, im ganzen also drei Doppelschwankungen aufgenommen. Zehn bis zwölf Doppelreize machten eine Versuchsreihe aus. Nach jeder Aufnahme wurde eine Pause von mehreren Minuten eingeschaltet, so dass der erste Reiz jedes Doppelreizes das Herz in einem Zustande traf, in dem irgendwelche Tätigkeitsspuren infolge vorhergehender Reize kaum anzunehmen waren.

Die Trommel öffnete bei einer Umdrehung zwei Kontakte, deren Abstand nach jedem Doppelreiz grösser genommen wurde. Wir suchten nämlich zu Beginn einer Versuchsreihe diejenige Reizdistanz aus, bei der noch keine Wirkung des zweiten Reizes zustande kam, und vergrösserten dieselbe sukzessive. Gleichzeitig mit der Aktionsstromkurve wurden die Signale für beide Reize und die Zeitkurve verzeichnet.

In beschriebener Weise angestellte Versuche ergaben überaus klare Resultate. Es geht erstens aus denselben hervor, dass bei Doppelreizen der Ventrikelspitze der zweite Aktionsstrom bei kurzem Reizintervall mit bedeutender Verspätung auftritt. Zweitens konnte man sich auch über eine andere, schon in früher beschriebenen Versuchen mehreremal beobachtete Erscheinung besser orientieren, nämlich über die Änderung der Form der stark verspäteten zweiten Aktionsstromkurve.

Was zunächst die Zunahme der Latenzdauer anbetrifft, so wollen wir wieder an der Hand einer Versuchsreihe die näheren Verbältnisse schildern. Aus der Tabelle II und der Graphik Fig. 4 sieht man, wie stark die Zunahme der Latenz sein kann. Wenn man in Betracht zieht, mit wie kleinen Schritten wir die Reizdistanz vergrösserten und dass nämlich die Reizdistanz, bei der noch keine Reaktion auf den zweiten Reiz bemerkbar ist, sich um einen sehr geringen Wert (0,039 Sek.) von der wirksamen Reizdistanz unterscheidet, so ist wohl anzunehmen, dass die im angeführten Versuch beobachtete Verspätung wohl nicht mehr weit von irgendeiner maximalen Verspätung sich befindet. Allerdings könnte man auch 


\section{Tabelle II.}

\begin{tabular}{c|c|c|c}
\hline $\begin{array}{c}\text { Nummer } \\
\text { des Doppelreizes }\end{array}$ & $\begin{array}{c}\text { Elektrische Latenz } \\
\text { des zweiten V.E. G. }\end{array}$ & Reizdistanz & $\begin{array}{c}\text { Summe der Zeit- } \\
\text { werte der vorher- } \\
\text { gehenden Kolonnen }\end{array}$ \\
\hline 1 & keine Reaktion & 2,399 & - \\
2 & keine Reaktion & 2,431 & - \\
3 & 0,364 & 2,470 & 2,834 \\
4 & 0,325 & 2,500 & 2,825 \\
5 & 0,273 & 2,535 & 2,808 \\
6 & 0,234 & 2,600 & 2,834 \\
7 & 0,208 & 2,626 & 2,834 \\
8 & 0,166 & 2,665 & 2,831 \\
9 & 0,140 & 2,717 & 2,857 \\
10 & 0,091 & 2,847 & 2,938 \\
11 & 0,078 & 2,990 & 3,068 \\
12 & 0,078 & 3,029 & 3,107 \\
13 & 0,078 & 3,185 & 3,263
\end{tabular}

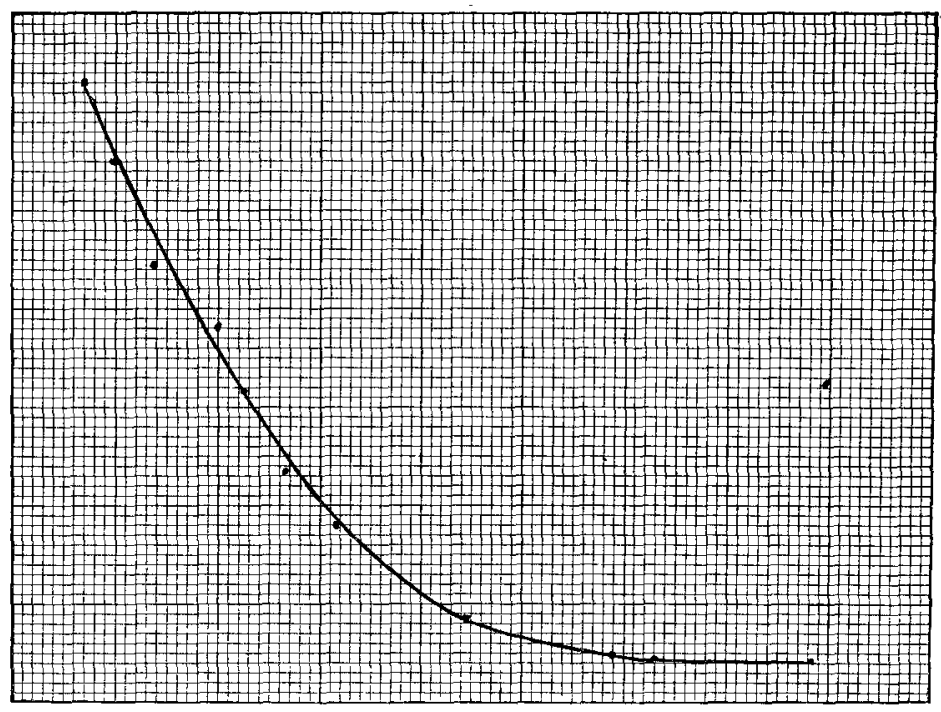

Fig. 4.

sagen, dass die Verspätung bei noch kürzerer Dauer mit Riesenschritten zunehmen würde und dass ein maximaler Grenzwert hier ins Unendliche versetzt werden müsste. Es ist aber praktisch sehr schwer, die Reizdistanz so zu wählen, dass die Verspätung des zweiten Aktionsstromes zwischen dem unendlichen Wert (d. h. bei unbeantwortetem Reiz) und einem nur um einigemal die normale Latenz übersteigenden Wert fällt. Dass man ganz bedeutende Verspätungen 
nicht beobachten kann, hat seinen Grund anderseits vielleicht auch darin, dass die verspäteten Schwankungen weniger steil (worauf wir noch später eingehen) und niedrig werden. Die Abnahme der Reaktionsfähigkeit des Herzens bej Doppelreizen mit zu kurzem Reizintervall geschieht also in zwei Richtungen - die zweite Schwankung tritt später auf, erscheint kleiner und gedehnter im Verlauf, wobei die ganze Schwankungsdauer stark abnimmt.

Der Wert 0,078 Sek. war in dem beschriebenen Versuch als normale Latenz anzusehen; durch das frühzeitige Eintreffen des zweiten Reizes ist die Latenz um etwa fünfmal grösser geworden.

Addiert man entsprechend die Werte der zweiten und dritten Kolonne, so bekommt man die Werte der vierten, die man als die irresponsive Periode im Sinne von Lucas auffassen kann. Wie man sieht, bleibt dieselbe nicht von gleichem Betrag im Bereiche der Reizdistanzen mit verspäteter Reaktion, sondern sie wird anfangs kleiner, erreicht ein Minimum und steigt dann weiter. In diesem Punkt befinde ich mich also auch bezüglich des Herzmuskels im Widerspruch mit K. Luc as, nach welchem die irresponsive Periode einen und denselben Wert behält.

Wir führen nun weiter einen Versuch mit sechs Doppelreizungen an, den wir mit entsprechenden Kurven illustrieren (s. Fig. 5). In der Abbildung ist nur der dem zweiten Reiz entsprechende Aktionsstrom wiedergegeben; würde man auch den ersten mit bineinnehmen, so würde die Figur zu lang werden (ausserdem ist die Reproduktion um zweimal gegenüber den Original verkleinert). Bei 1, Fig. 5, fällt der zweite Reiz vor der Zacke $T$ der ersten Schwankung; er bleibt ohne Erfolg - man sieht nur eine kleine Senkung, von einer Stromschleife herrührend. Rückt der Reiz weiter vom ersten weg, sa wird er manifest. Auf der Höbe des T-Gipfels das Herz treffend bewirkt der zweite Reiz (s. 2, Fig. 5) einen Aktionsstrom mit einer ganz bedeutenden Verspätung. Je grösser die Reizdistanz wird, um so kleiner wird die Verspätung, bis sie schliesslich in den Doppelreizen 5 und 6 fast ganz verschwindet. Gleichzeitig mit der $\mathrm{Zu}$ nahme der Reizdistanz wächst auch, wie in der Regel, die Zeitdauer der ganzen zweiten Schwankung. Die entsprechenden Zahlenwerte sind in der Tabelle III zusammengestellt.

Wir erwähnten früher, dass bei kurzem Reizintervall, wenn die zweite Schwankung mit bedeutender Verspätung auftritt, die Form der entsprechenden Aktionsstromkurve verändert erscheint. 
Über die Latenz der elektr. Reaktion des Froschherzmuskels etc.

Tabelle III.

\begin{tabular}{c|c|c|c}
\hline $\begin{array}{c}\text { Nummer } \\
\text { des } \\
\text { Doppelreizes }\end{array}$ & $\begin{array}{c}\text { Elektrische } \\
\text { Latenz } \\
\text { der zweiten } \\
\text { Schwankung } \\
\text { Sek. }\end{array}$ & Sek. & Reizdistanz \\
\hline & $\begin{array}{c}\text { Irresponsive } \\
\text { Periode }\end{array}$ \\
\hline 1 & $0, \overline{178}$ & 2,767 & - \\
3 & 0,073 & 2,903 & 3,081 \\
3 & 0,062 & 3,105 & 3,043 \\
4 & 0,057 & 3,199 & 3,167 \\
5 & 0,057 & 3,281 & 3,256 \\
6 & & &
\end{tabular}

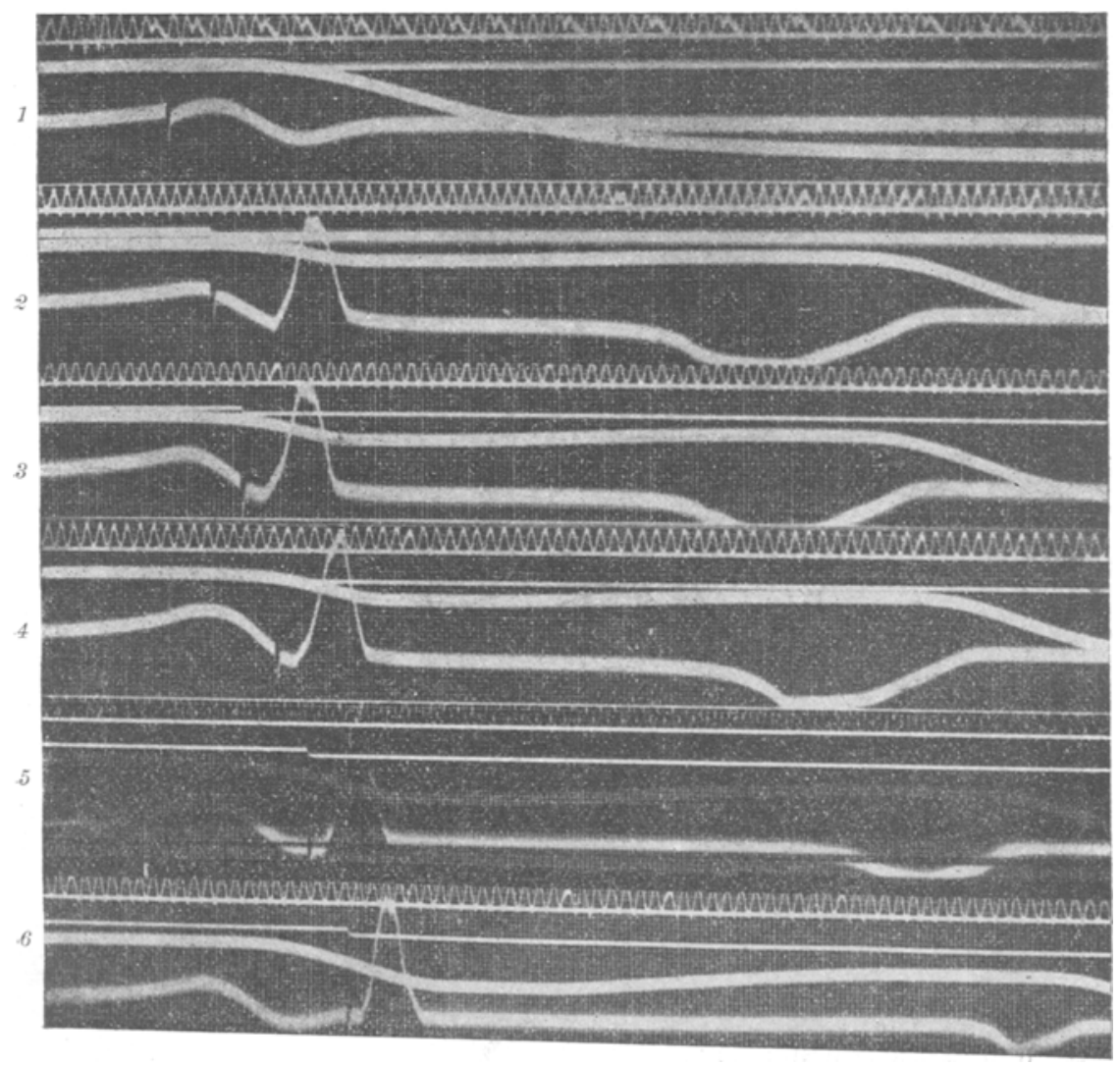

Fig. 5. Die Originalkurven sind zweimal grösser und beginnen mit dem ersten Aktionsstrom, von dem hier nur die Zacke $T$ zu sehen ist. Jede der sechs Aufnahmen weist von oben nach unten auf: Zeitmarken $(0,04$ Sek.), Reizsignal 1 , Reizsignal 2, Kontraktionskurve, Aktionsstrom. 
Die Zacke $R$ verliert ihre schlanke Gestalt, sie wird gedehnt. Dieses Verhalten ist sehr schön in der angeführten Fig. 5, namentlich beim Vergleich der $R$-Zacken in 2 und 3 gegenüber 6 , zu sehen (in 5 war während der Rotation der Trommel durch ein Ver-
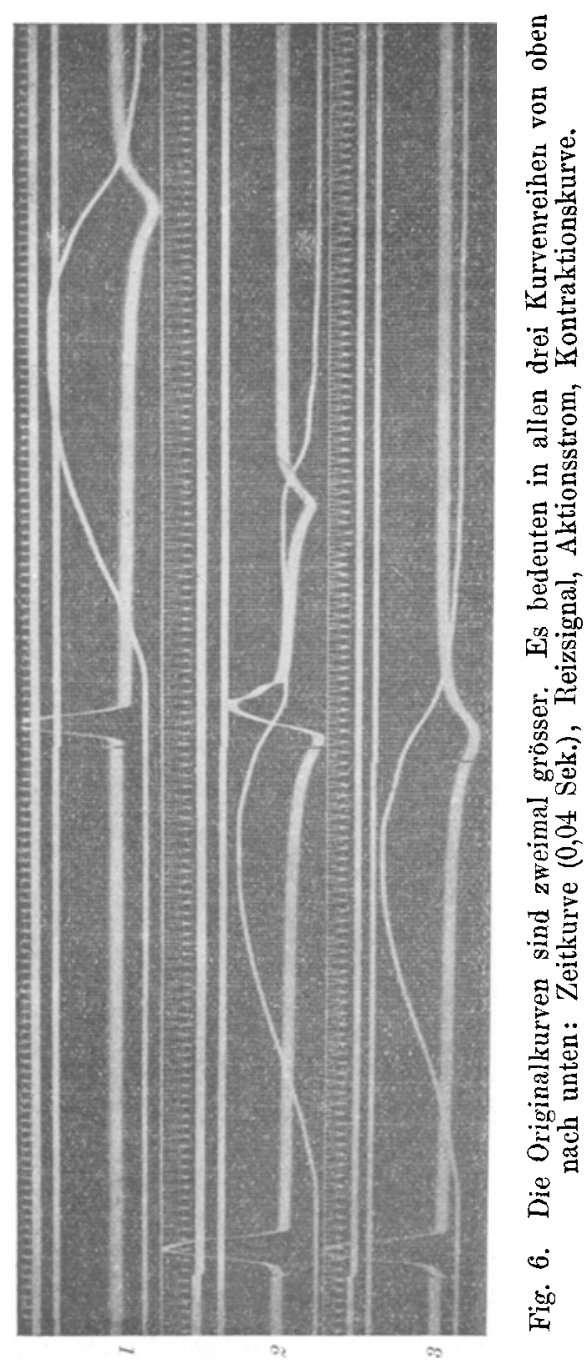
sehen die Nebenschliessung des Galvanometers nicht geöffnet, weshalb der Doppelreiz wiederholt und noch einmal auf demselben Papierstreifen aufgenommen wurde). Entsprechend der gedehnten Form ist die Kuppe der $R$-Zacke in 2 abgerundet; meistens beobachtet man manche Unebenheiten im Verlaufe der verspäteten zweiten Kurve.

Diese Veränderung der Form der zweiten Schwankung ist gut an der Hand der Fig. 6 , die überhaupt einige Hauptmomente der ganzen Erscheinung der Aktionsströme bei Doppelreizen des Herzens gut illustriert, zu sehen. Bei der ersten (1, Fig. 6) Rotation der Trommel wird nur der Kontakt des zweiten Reizes geöffnet; man erhält also ein Ventrikelelektrogramm bei der alleinigen Wirkung des zweiten Reizes, das die gewöhnliche Form des zweiphasigen Stromes mit den $R$ - und T-Zacken aufweist. Die Latenz ist auf Grund des Reizmarkierers sowie der Stromschleifenzacke abzulesen; sie beträgt 0,032 Sek. Die zweite (2, Fig. 6) Umdrehung öffnet beide Kontakte, wobei die Reizdistanz (2,08 Sek.) so gewählt ist, dass der 
zweite Reiz den Herzmuskel im Momente trifft, wo die T-Zacke geschrieben wird. Die elektrische Reaktion erscheint verspätet im Vergleich mit der Kurve bei alleiniger Wirkung des zweiten Reizes; die Latenz der zweiten Schwankung beträgt jetzt 0,054 Sek. Es ist bemerkenswert, dass die mitregistrierten mechanischen Kurven der Herztätigkeit keine Verspätung erkennen lassen, ja im Gegenteil würde man auf Grund der Kontraktionskurve eher eine Verfrühung als eine Verspätung annehmen dürfen; es ist an diesem Beispiele gut zu ersehen, dass die betreffende Frage über die Latenz bei Doppelreizen auf Grund der Herzkontraktionskurven nicht aufgeklärt werden kann. Die Dauer der elektrischen Aktion in 2, Fig. 6, ist etwa zweimal kürzer wie in 1. Bei der dritten Umdrehung 3, Fig. 6 , war die Reizdistanz um 0,048 Sek. kürzer genommen, und der zweite Reiz blieb unbeantwortet.

Die Form der zweiten Stromkurve beim wirksamen Doppelreiz in 2 , Fig. 6 , ist ganz bedeutend verändert. Es sei darauf aufmerksam gemacht, dass die Formänderung sich hier auf eine Stromkurve bezieht, die durchaus nicht extrem verspätet beginnt; man kann deshalb sagen, dass in der Tat die Verspätung und die Formänderung der Aktionsstromkurven zwei verschiedene, aber in ihrer Bedeutung auf gleicher Stufe stehende Seiten einer und derselben Erscheinung, nämlich der veränderten Reaktion des Herzens nach kurzem Reizintervall, darstellen; einmal tritt die eine, ein anderes Mal die andere Seite mehr in Szene.

Was die Form der $R$-Zacke der zweiten Schwankung in 2, Fig. 6 , anbetrifft, so ist sie abnorm gedehnt. Entsprechend der Dehnung ist die Kuppe der $R$-Zacke abgerundet; am absteigenden Teil sieht man eine Unebenheit. Die Höhen der Zacken $R$ und $T$ sind verkleinert im Vergleich zur Höhe derselben beim ersten Reiz in 2 und 3 und dem zweiten in 1 der Fig. 6.

Was diese Veränderung der Form bedeutet und wie dieselbe zustande kommt, ist schwer zu sagen. Die Ursache der gedehnten Form könnte man sich vielleicht in folgender Weise erklären: Der zweite Reiz, der nach der möglichst kurzen Zeitdistanz nach dem ersten durch das Herz geschickt wird, findet noch nicht sämtliche Muskelfasern reaktionsfähig: die einen reagieren schon, die anderen sind noch refraktär, aber im nächsten Moment sind sie ebenfalls für den Reiz empfänglich usw. Die Kurve des Aktionsstromes würde nicht den normalen Gang des Erregungsablaufes, sondern vielmehr 
des Erregungsaufwachens darstellen. Was anderseits die Verspätung selbst anbetrifft, so lässt sich auch nicht ohne weiteres die Möglichkeit negieren, dass die gedehnte Form und namentlich der gedehnte Beginn der zweiten Schwankung die Verspätung, wenn nicht ganz, so doch zum grossen Teil vortäuschen kann, wie ich es an einer anderen Stelle bezüglich des quergestreiften Muskels dargetan habe ${ }^{1}$ ).

Es sei hier noch darauf hingewiesen, dass in sämtlichen Publikationen über Elektrokardiogramme, in welchen man Aktionsstromkurven von Extrasystolen findet, sei es in pathologischen Fällen von Menschen oder sei es von Säugetieren bei Experimenten, meistens die Zacke $R$ in einer Weise verunstaltet sich erweist, die sehr an die eben mitgeteilte Veränderung der $R$-Zacke der zweiten Schwankung bei Doppelreizen erinnert.

Wenn nun zum Schluss jemand die Frage vorlegen würde, ob man die beschriebenen Versuche als eine Bestätigung der Behauptung von K. Lucas über das verspätete Auftreten des zweiten elektrischen Effektes bei Doppelreizen mit kurzem Reizintervall betrachten kann, so hätte man meiner Meinung nach folgendermaassen antworten dürfen: Unzweifelhaft ist die Behauptung von $\mathrm{Lu}$ cas richtig, wenn auch nicht im ganzen Umfange. Zweifelhaft scheinen mir dagegen die Gründe, die diesen Autor zur richtigen Behauptung führten.

Jetzt noch mehr wie früher scheint es mir wahrscheinlich, dass die in den Lu c a s' schen Figuren (12 und 13 der zitierten Abhandlung) auftretende Verspätung, die das Zehnfache der normalen Latenz beträgt (eine Verspätung, die ich in einem so grossen Betrage nicht beobachtete), durch Mitreizen des Vorhofs und Überleiten des Reizes erklärt werden kann, denn: 1. fällt der diese ausserordentliche Verspätung bewirkende Reiz gar nicht früh genug, er trifft vielmehr das Herz ganz nach Beendigung der Schwankung, weshalb die Verspätung vom Standpunkte meiner Versuche ganz unmotiviert erscheint, und 2. besitzt die so ungewöbnlich stark verspätete zweite elektrische Aktion in der Figur von $L u c a s$ eine der ersten Schwankung durchaus ähnliche Form, was wiederum meinen Erfahrungen über die Entstellung der verspäteten Stromschwankungen widerspricht.

1) A. Samojloff, Über die Verspätung des zweiten Aktionsstromes usw. Arch. f. d. ges. Physiol. Bd. 143 S. 453. 1912. 
Der Inhalt der vorliegenden Untersuchung kann kurz in folgenden Worten resumiert werden:

1. Die elektrische Latenz hängt wesentlich von der Reizstärke ab; bei starkem Reiz ist sie kürzer, bei einem schwachen länger.

2. Bei stärkeren Reizen hängt die Latenz von der Phase der Herztätigkeit, auf die der zweite Reiz des Doppelreizes fällt, ab.

Wenn der Reiz ganz nach Ablauf der Schwankung den Ventrikel trifft, so ist es kaum möglich, eine Änderung der Latenz zu verzeichnen; trifft dagegen der Reiz früher ein, etwa am Gipfel oder auch im zweiten Teil der T-Zacke, so wird die Latenz grösser, wobei dem früher eintreffenden Reize eine grössere Dauer der latenten Periode entspricht. Der grösste beobachtete Wert der Verspätung betrug etwa das Fünffache der Anfangslatenz.

3. Die bei kurzem Reizintervall stark verspätete Herzaktion resultiert einen Aktionsstrom, dessen Form stark verändert erseheint; der Verlauf der elektrischen Kurve, namentlich der der $R$-Zacke, wird bei geringerer Höhe gedehnt und unregelmässig.

Als die obige Untersuchung abgeschlossen war, erschien die Abhandlung von W. Trendelenburg „Über die zeitliche Beziehung der Refraktärphase des Herzens zu seinem Aktionsstrom" (Dieses Arch. Bd. 144, 3, 39), in welcher wir folgende Stelle, die unser Thema berührt, finden: „Fraglich erscheinen mir weiterhin Werte, die Lucas für das Froschherz angibt. Wurden zwei Reize angewendet, so war die Latenz des elektrischen Vorganges bei dem zweiten beträchtlich länger wie bei dem ersten; z. B. in einem abgebildeten Falle (Fig. 13 der Abhandlung von Lucas) etwa 0,4 gegen 0,02 Sek. (ungefähre Scbätzung nach der Figur). Obwohl ich mich in meinen Versuchen mit dem zweiten Reiz stets an der Zeitgrenze der Erfolgsmöglichkeit hielt, habe ich doch derart lange Latenzen nie beobachtet. Allerdings ist die Latenz bei möglichst frühzeitig einfallendem zweiten Reiz häufig verlängert, wie z. B. Fig. 2 zeigt, in welcher sie etwa 0,2 Sek. gegen 0,1 Sek. beim. ersten Reiz beträgt. Dies ist aber auch der grösste von mir beobachtete Wert.“ Also auch für W. Trendelenburg waren die Angaben von K. Lucas fraglich, wie für mich. Wenn man die Doppelreizungen so vornimmt, wie es bei $L$ ucas der Fall ist, d. h. wenn der zweite Reiz ganz nach Ablauf der ersten Schwankung einsetzt, so lassen 
sich keine derartigen Verspätungen beobachten, wie die von Lucas behaupteten; darin bin ich mit W. Trendel en burg einverstanden. Dass anderseits Trendelenburg Latenzen von grösserer Dauer nicht fand, ist einfach daraus zu erklären, dass er absichtlich mit sehr schwachen Reizen arbeitete und also nicht früh genug den zweiten Reiz mit Erfolg einwirken lassen konnte, während wir eben, um möglichst kurz die Zeitdistanz der wirksamen Reize nehmen zu können, absichtlich von stärkeren Reizen Gebrauch machten. 